\title{
Rapid HIV Testing in Al Buraimi Governorate Sultanate of Oman: A Cross Sectional Study
}

\author{
Saad Rashid Saud Al Alawi', Muhammad Muqeet Ullah², Ahmed Yar Mohammed Dawood Al Balushi², \\ Rajeev Kashyap ${ }^{3} \&$ Vandita Kailas Patil ${ }^{4}$ \\ ${ }^{1}$ Regional HIV Counselor, Al Buraimi Hospital, Al Buraimi Governorate, Ministry of Health, Oman \\ ${ }^{2}$ Directorate of Communicable Diseases Surveillance and Control, Directorate General of Health Services, Al \\ Buraimi Governorate, Ministry of Health, Oman \\ ${ }^{3}$ Medicine Department, Al Buraimi Hospital, Al Buraimi Governorate, Ministry of Health, Oman \\ ${ }^{4}$ Obstetric and Gynecology Department, Al Buraimi Hospital, Al Buraimi Governorate, Ministry of Health, Oman \\ Correspondence:Saad Rashid Saud Al Alawi' Regional HIV Counselor, Al Buraimi Hospital, P.O..Box: 8, Postal \\ Code 512, Al Buraimi Governorate, Ministry of Health, Sultanate of Oman. E-mail: saadalawi14@gmail.com
}

Received: August 6, 2018 Accepted: September 30, 2018 Online Published: October 24, 2018

doi:10.5539/gjhs.v10n11p153 URL: https://doi.org/10.5539/gjhs.v10n11p153

\begin{abstract}
Background: Due to one of the highest HIV prevalent province in Oman, Voluntary Counselling Testing (VCT) services were integrated in health system of Al Buraimi Governorate, Oman since December 2014.
\end{abstract}

Aim and Objective: The study aimed to analyze the situation on HIV rapid testing in Al Buraimi Governorate with objective to identify client's risk factor among common age group and gender.

Methodology: Cross sectional retrospective study was conducted in Al Buraimi Governorate, Oman among 1412 registered adult VCT clients, unbooked pregnant women with no documented HIV status at the time of delivery or abortion at Buraimi Hospital and clients during outreach community awareness activities on HIV. Retrospective analysis was performed for 3 years from December 2014 to December 2017 on parameters like Age, gender, nationality, risk factors, outcome and place of testing (Hospital and Outreach) using standard national client information form. Descriptive statistics was applied in Microsoft excel and SPSS version 24. Clients' personal information and confidentiality of the record was maintained during entire study period with approval from regional research and ethical review committee.

Results: Of 1412 clients for Rapid Diagnostic Tests (RDTs), 990 (70.1\%) were females and 422 (29.9\%) were males with mean age 27.13 \pm 7.02 . Majority, 806 (57.1\%) RDTs were in outreach followed by $470(33.3 \%)$ and 136 (9.6\%) at Maternity ward and Couselling Clinic respectively. Clients included 1294 (91.6\%) Omani nationals and $118(8.4 \%)$ non-Omani nationals. The risky behavior was found to be $126(9 \%)$ among clients with heterosexual contributes $65(4.6 \%)$. Among all risk factors, male clients contributed 124 (8.7\%) with common age group 26-35 years $56(3.9 \%)$.

Conclusion: RDTs were utilized mainly during outreach activities that highlight the need of promoting VCT facilities in the clinical setting as services are available $24 / 7$ through hotline. This baseline study would facilitate to develop plan for client's risk reduction.

Keywords: rapid diagnostic tests, human immunodeficiency virus, voluntary counselling and testing

\section{Introduction}

HIV continues to be a major global public health issue. 36.9 million people globally were living with HIV in 2017. There were 1.8 million new HIV infections, compared to 3.4 million in 1996. New HIV infections have been reduced by $47 \%$ since the peak in $1996.75 \%$ of all people living with HIV knew their HIV status in 2017. AIDS-related deaths have been reduced by more than 51\% since the peak in 2004. 940, 000 people died from AIDS-related illnesses in 2017. Every week, around 7000 young women aged 15-24 years become infected with HIV. The risk of acquiring HIV is 27 times higher among men who have sex with men; 23 times higher among people who inject drugs; 13 times higher for female sex workers; 12 times higher for transgender women.Despite challenges, new global efforts have meant that the number of people receiving HIV treatment has increased 
dramatically in recent years, particularly in resource-poor countries ((UNAIDS 2017-18 fact sheet, Global HIV and AIDS statistics).

HIV testing services are the gateway to prevention services and life-saving treatment and care. Between 2010 and 2014 nearly 600 million adults (ages 15+) reportedly received HIV testing services in 122 low- and middle-income countries in this period. Approximately 150 million children and adults in 129 low- and middle-income countries reportedly received HIV testing services in 2014. Approximately 3 million children and adults tested HIV-positive according to 81 reporting low- and middle-income countries in 2014 . Nearly $70 \%$ of adults receiving HIV testing services are women. Much testing is occurring in antenatal care settings, even in low and concentrated epidemic settings. Across all WHO regions, more than $60 \%$ of adults testing HIV-positive were women; outside the WHO African Region, however, those who tested HIV-positive were more likely to be adult men (WHO, July 2015 Fact Sheet).

UNITAID and the World Health Organization (WHO) in July 2016 released the second edition of the landscape report on HIV self-testing which shows that 16 countries have adopted HIV self-testing policies, while many others are currently developing them. The report finds that the market for HIV self-testing tools is clearly growing - a trend that could help to meet global demand which is estimated to reach at least 4.8 million HIV rapid diagnostic tests by 2018. In the long run, HIV self-testing could expand testing in countries and help to meet United Nations targets, including diagnosing 90 percent of all people with HIV by 2020 (WHO, UNITAID 2016).

The WHO classifies the HIV epidemic in Oman as a low-prevalence epidemic. The first case of HIV in Oman was detected in 1984. At the end of 2016, 2780 HIV/AIDS cases were reported among Omanis with 1096 deaths since 1984. Of all reported cases, 1684 Omanis were alive at the end of 2016 with 63\% PLHIV were assessed with at least one CD4 count or viral load in the year 2016. At the end of 2016, 1183 PLHIV are on ART, 788 were males and representing $66.6 \%$ of male PLHIV and 395 females representing $33.3 \%$ of Female PLHIV (Annual Health Report, Chapter 8, $2016 \mathrm{MOH}$ Oman). Heterosexual transmission accounted for the majority of infections (51.1\%) followed by homosexual-bisexual (13.9\%), mother-to-child 9 (5.5\%), injection-drug use (4\%) and blood transfusion (2.8\%). In 2014, HIV infection was reported among 146 non-nationals. In Oman, male population contributes to the majority (70\%) of PLHIV(HIV managment in Oman, 2015).

Al Buraimi Governorate which is situated in the North West corner of the Sultanate bordering UAE with estimated total mid-year 2016 population 111,394 (Omani, 48\% and Expatriate 52\%). Al Buraimi population constitutes $2.5 \%$ of total national population (4,414,051), (Annual Health Report, Chapter 1: $2016 \mathrm{MOH}$ Oman).

In 2014, National AIDS Program integrated VCT services in health care system at three governorates of Oman (Muscat, Al Buraimi and North Batinah ) due to high prevalence. According to Ministry of Health Annual report 2016, highest prevalence was found to be in Al Buraimi Governorate, 139 alive Omani cases (prevalence 262/100,000) followed by Muscat Governorate 664 alive Omani cases (prevalence 130/100,000) and North Batinah Governorate, 377 alive Omani cases (prevalence 79/100,000) respectively (Annual Health Report, Chapter 8, $2016 \mathrm{MOH}$ Oman).

VCT services at Al Buraimi Hospital was established in December 2014 with specific Hotline Number working 24/7 and is headed by Regional HIV Counselor under supervision of Director of Al Buraimi Hospital and Directorate of Communicable Disease Surveillance and Control, DGHS Al Buraimi Governorate Oman. The aim of VCT introduction in Oman is to provide unique service in which the client makes the conscious decision to actively seek HIV testing with no external coercion. In view of importance of RDTs, this baseline study would facilitate to reach out high risk population and to offers an entry point for early care and support for those infected with HIV and Prevention of Mother to Child Transmission (PMTCT).

\section{Methodology}

\subsection{Design and Study Population}

Cross sectional study was conducted among 1412 adult clients who were registered (since start of VCT service) during span of 3 years from December 2014 to December 2017 in Al Buraimi Governorate, Oman.

\subsection{Incusion Criteria}

The study included VCT clients consulted through specific hotline number which is made available 24/7 in counseling clinic headed by regional HIV counselor at Buraimi Hospital, unbooked pregnant ladies with no HIV status at time of delivery in delivery suite or ladies presented with abortion and clients who were consulted during outreach community awareness activities on HIV during 3 years period from December 2014-2017. 


\subsection{Exclusion Criteria}

The exclusion criteria included clients who were aged less than 12 years, clients with no record during 2014-2017 and clients who were tested and diagnosed initially through routine blood test (ELISA and Western Blot).

\subsection{Data Collection and Analysis}

Retrospectively data was reviewed during 3 months from standard national form for the client aged 12 years or more who underwent RDTs from December 2014 to December 2017 and were interpreted by non-laboratory personnel. RDTs results were available within 20-30 minutes as accurate as ELISA/EIA assays and were interpreted as positive (reactive), negative (non-reactive), or invalid. Clients underwent a pretest counselling and post test counselling session after taking consent from clients. Clients' information was recorded in standard national client form.

\subsection{Analysis}

Retrospective data analysis was performed using descriptive statistics in Microsoft excel and SPSS version 24 on parameters like Age, gender, nationality, risk factors, outcome of test and place of testing (Couselling clinic, Maternity ward and Outreach).

\subsection{Ethical Consideration}

Clients' personal information and record was maintained confidential during entire study period. The study was approved by Regional Research and Ethical Review Committee Directorate General of Health Services Al Buraimi Governorate, Oman.

\section{Results}

The result of this cross sectional study after retrospectively record review of 1412 clients (including VCT clients, clients with un-booked pregnant ladies and outreach clients) showed that 990 female clients and 422 male clients were tested using RDT kits. Descriptive analyses showed that majority of the clients were in between age group 15-35 years. Mean age was 27.13 7.02. Omani National comprised of 1294 (91.6\%) and Non Omani $118(8.4 \%)$. Of 1412 clients, $126(9 \%)$ identified with risk factors during pre and post test counselling while $1286(91 \%)$ were having no risk factors. Among 126 clients with risk factor heterosexual comprised of 65 (4.6\%), I/V drug 22 (1.6\%) and Homosexual 19 (1.3\%). Clients with bisexual comprised of 18 (1.3\%). 1 client was tested positive by RDT and was linked to care and treatment after confirming the test through confirmatory assays by ELISA and Western Blot.(See Table 1).

Table 1. Characteristics of the RDTs clients ( $\mathrm{N}=1412)$

\begin{tabular}{llll}
\hline S.No & Parameters & Number (n) & Percentage (\%) \\
\hline Age group & $15-25$ & 733 & $51.9 \%$ \\
Mean & $26-35$ & 498 & $35.3 \%$ \\
$27.13 \pm 7.02$ & $36-45$ & 170 & $12 \%$ \\
& $46-55$ & 11 & $0.8 \%$ \\
\hline Sex & Male & 422 & $29.9 \%$ \\
& Female & 990 & $70.1 \%$ \\
\hline Nationality & Omani & 1294 & $91.6 \%$ \\
& Non Omani & 118 & $8.4 \%$ \\
\hline Risk Factors & I/V drug & 22 & $1.6 \%$ \\
& Heterosexual & 65 & $4.6 \%$ \\
& Homosexual & 19 & $1.3 \%$ \\
& I/V and Homosexual & 2 & $0.1 \%$ \\
& Heterosexual and Homosexual & 18 & $1.3 \%$ \\
& None & 1286 & $91.1 \%$ \\
\hline Outcome & Positive & 1 & $0.1 \%$ \\
& Negative & 1411 & $99.9 \%$ \\
\hline
\end{tabular}


Outreach activities were utilized more for RDTs as compared to clinical setting area. Outreach comprised of 806 (57.1\%) followed by Maternity ward 470 (33.3\%) and Counselling clinic 136 (9.6\%) clients (See Figure 1).

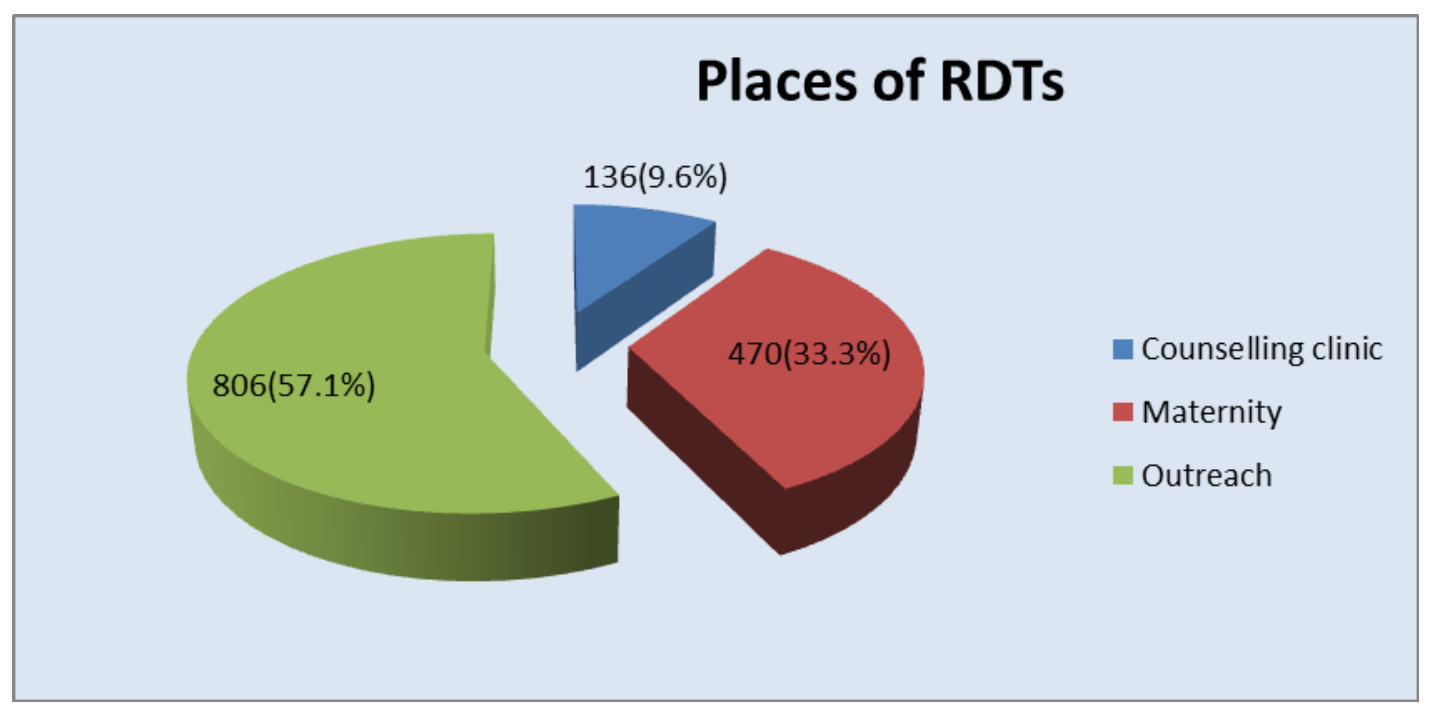

Figure 1. Places of RDTs ( $\mathrm{n}=1412)$

Clients during pre and post test counseling were evaluated for their risk factor. It was shown that Heterosexual is the most common risky behavior $65(4.6 \%)$ prevalent among the clients with majority risky behaviors in age group between 15-35 years and male predominance (exposed to risky behavior) among 1412 RDTs clients. (See Figures $2 \& 3)$.

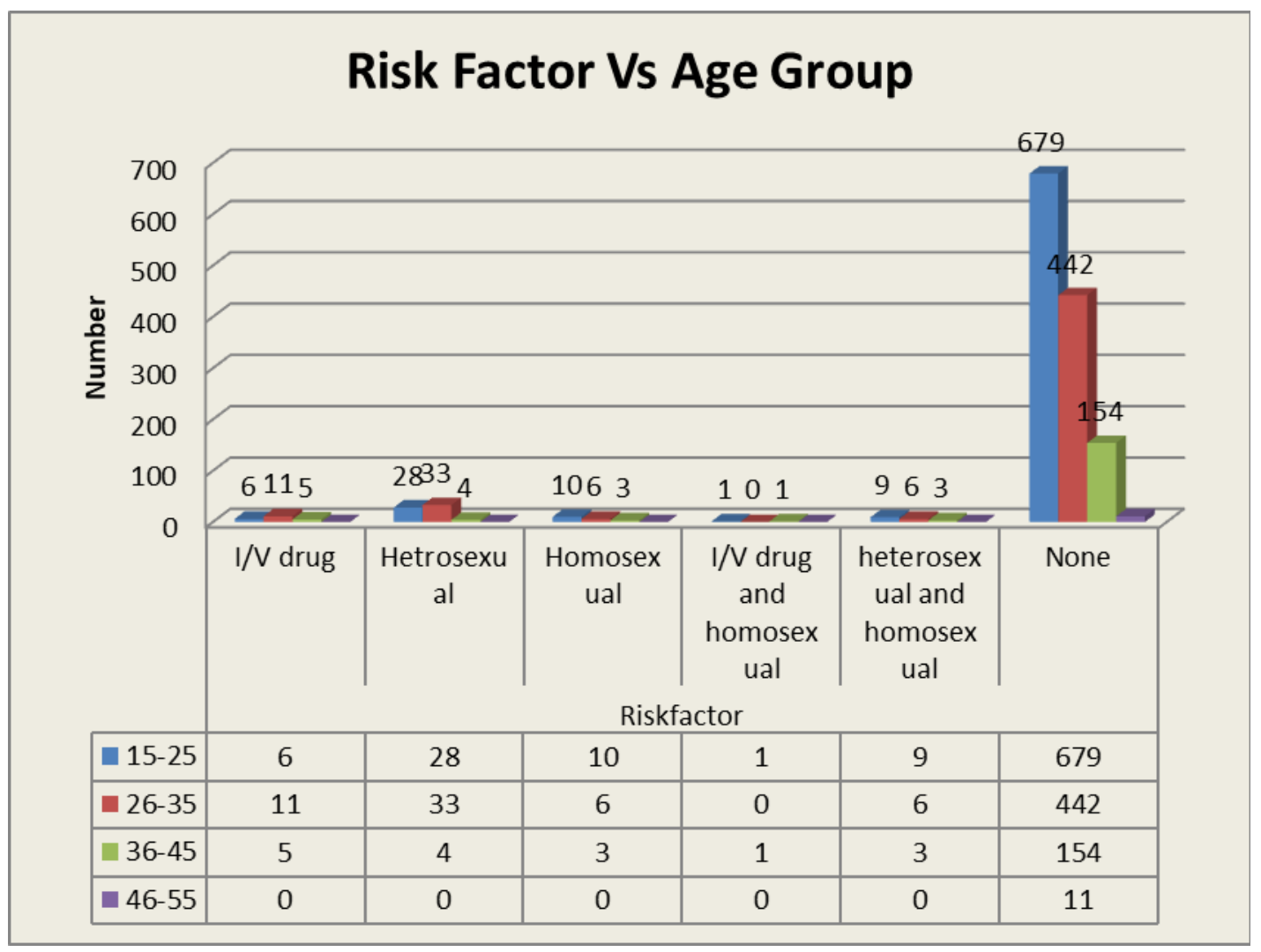

Figure 2. Risk factor among age groups $(\mathrm{N}=1412)$ 


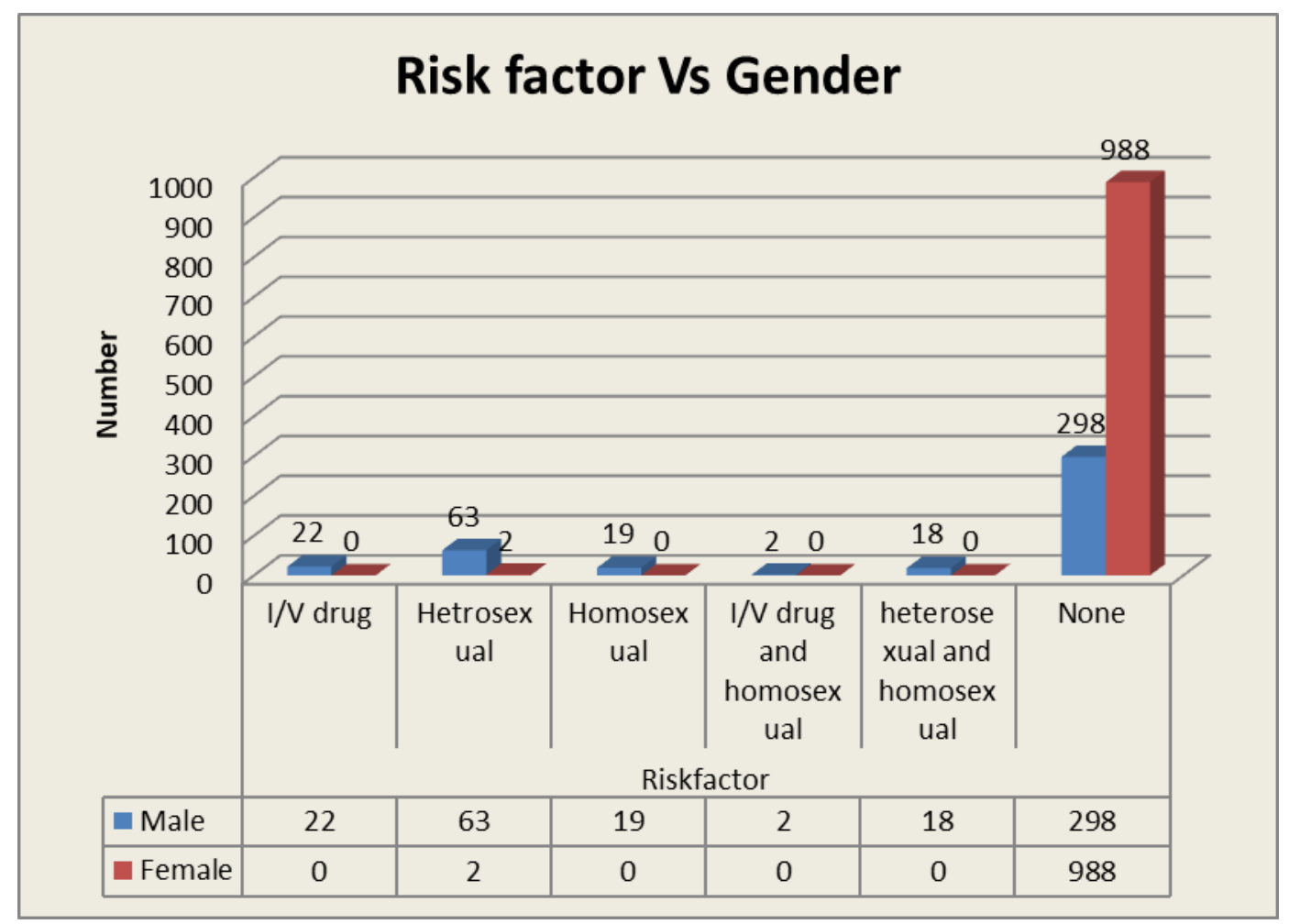

Figure 3. Risk factor among gender $(\mathrm{n}=1412)$

\section{Discussion}

AIDS control programme in Oman was established in 1987 and was considered as one of the health programs since the fourth Five-Year Health Development Plan starting (1991). Since then the NAP has continued to scale-up its services to people living with HIV (PLHIV). One of the Strategies of NAP in prevention of HIV transmission is to increase access to and utilization of HIV testing and counseling (HTC) services. Rapid Diagnostic, a fourth generation screening assay and is utilized for VCT clients, during awareness campaign, for patients admitted to ICU with suspected HIV infection, Occupational exposure to HIV infection and pregnant women who have no documented HIV status at the time of delivery. Rapid results are available within 20-30 minutes and are almost as accurate as ELISA/EIA assays. HTC is the entry point to all HIV prevention and treatment services. It can be delivered by different approaches like Voluntary Counselling and Testing (VCT) and Provider-initiated testing and counselling (PITC).

Generally, HIV serologic assays are classified as screening (or first-line) assays and supplemental (or confirmatory) assays. Screening assays are fourth-generation screening assays which are highly sensitive due to incorporation of p24 antigen. There are 2 techniques available for screening:

a. ELISA/EIA (enzyme-linked immunosorbent assay/enzyme immunosorbent assay)-Are commonly used at facilities with high output on a daily basis, e.g. blood banks and tertiary/central laboratories. It contains antigens from both HIV-1 and HIV-2 and are therefore able to detect infections with either of these viruses. It needs sophisticated laboratory equipment and takes 3 hours, at minimum, to yield results. It is reported as positive (reactive) or negative (non-reactive) test results.

b. Rapid Diagnostic Tests (RDTs)-also known as rapid tests is a much easier to be used and interpreted by non-laboratory personnel allowing testing, counseling and referral to be done in one visit. Rapid results are available within 20-30 minutes and are almost as accurate as ELISA/EIA assays. It is interpreted as positive (reactive), negative (non-reactive) or invalid.

As recent national policy published in the manual of HIV Management 2015 by Ministry of health Oman, the diagnosis of HIV infection must be confirmed by a laboratory using different validated assays. An individual can approach HIV diagnosis through different models of HIV testing and counseling (including VCT) services. HTC is intended to allow people to make informed decisions regarding the knowledge of their HIV status and the implications of those decisions. A range of diverse models and approaches of HTC can be used such as Voluntary 
counseling and testing (VCT), Provider-initiated testing and counseling (PITC) and Community-based approaches (HIV management in Oman, 3rd edition 2015 MOH Oman).

Hermez J, published literature on HIV testing policies and practices in the 22 countries of the Eastern Mediterranean Region, including surveillance, monitoring and evaluation reports. Field observations were conducted in four countries (Oman, Pakistan, Sudan and Egypt). Of reported diagnostic HIV tests conducted in the Eastern Mediterranean Region from 1995 to 2008, 59.3\% were carried out on migrant workers. Only 4.0\% were carried out on key populations at higher risk for HIV and $8.1 \%$ were conducted in sexually transmitted infection, tuberculosis and antenatal care services. It was concluded that although policies in the Eastern Mediterranean Region include a mix of mandatory and voluntary HIV testing, mandatory testing predominates, especially for migrant and foreign workers and key populations at higher risk of HIV,( Hermez, Petrak, Karkouri, \& Riedner, 2010). The current study of Al Buraimi Governorate Oman reviewed the record of 3 years for RDTs (high risk groups) which covered both clinical setting as well as outreach. The VCT services at counselling clinic comprised only $136(9.6 \%)$ of total 1412 clients which were utilized through hotline.

In Oman, mandatory blood testing predominates as national policy for high risk population including migrant and foreign workers. Also, mandatory testing is done as routine for all pregnant ladies registered in ANC clinics all over Oman. Due to high prevalence of HIV cases in 3 regions of Oman (Buraimi, Muscat and North Batinah) ministry had launched VCT services and integrated into health service. These VCT services were established at counselling clinics which run through hotline. They are utilized during outreach activities during community awareness programs on HIV. So, RDTs services are not limited to clinical setting only.

A two-stage sample design was conducted among 9,865 households in Kenya among women aged 15-49 years and determinants were analyzed using VCT services for females using data from the Kenya Demographic and Health Survey of 2003. The analysis focused on women since they were believed to be at a higher risk of infection as evidenced by women HIV incidence which is twice that of men in Africa, (Namzzi, 2003). The current study in Al Buraimi Governorate focused on adult clients who utilized the RDTs from December, 2014 to December, 2017 through hotline in counseling (VCT) clinic, RDTs for unbooked pregnant ladies with no HIV status at the time of delivery or abortion as well as for RDTs during outreach activity. About 1412 clients included both gender from age group 15-55 years utilized the services of VCT.

The VCT services utilized among women in Kenya was reasonably low despite the enormous benefits. Barriers to use of VCT services by women due to limited gender roles and fear of partner's reaction need to be addressed, (Namazzi, 2003) whereas current study in Al Buraimi Governorate, Oman showed female predominance 990 (70.1\%) for RDTs utilization as compared to male 422 (29.9\%).

In June 2012, USAID published fact sheet on existing evidence from rigorously evaluated interventions to prevent HIV transmission in developing countries. The findings presented in the fact sheet come from a meta-analysis of 17 studies with majority took place in a clinical setting. The meta-analysis results from fact reports of USAIDS indicated that participants receiving VCT were more likely to report reducing their number of sexual partners than those who did not receive VCT. Although VCT did not have a significant overall effect on condom use, people living with HIV who received VCT reported an increase in condom use compared to people living with HIV who did not receive VCT, (USAID-usebale results. Fact sheet June 2012), whereas this current study of ours was a cross sectional retrospective in nature focusing on the record of clients consulted through hotline and outreach community activities. Also, this study aimed to determine risk factors among different age group and gender which were explored during pre and post counseling session. The risk behavior was found to be $126(9 \%)$ among all clients with risk factor was most common in age group 26-35 years.

In recent years, especially in resource limited settings the primary methodology for HIV testing has shifted from enzyme linked immunosorbent assay (ELISA) to rapid diagnostic tests (RDTs). They can be used as first line followed with ELISA testing and Western blot for confirmation. In a study conducted in North India tertiary care hospital VCT facility considered performance of RDTs in comparison to ELISA is suboptimal after evaluating 787 sera of the patients. False negatives by RDTs increase the proportion of HIV reactive individuals receiving negative reports, false positives by RDTs are a matter of ethical concern. The study concluded that RDTs were fare poorly compared to ELISA as screening assays and that reactive results by RDTs need to be confirmed by western blot for a positive serodiagnosis of HIV infection, (Mehra, Bhattar, Bhalla, \& Rawat, 2014). In Oman, VCT services were introduced in three high prevalent region including Al Buraimi Governroate with main focus on high risk groups. The reactive results by RDTs are to be confirmed by ELISA follwed by western blot test.

An equity-focused systematic review ( 8 randomized controlled trials and 5 observational) study was conducted from 1st January 2001 to 5th June 2014 by Pottie.K, et.al to assess the effects of rapid voluntary counselling and 
testing (VCT) for HIV on HIV incidence and uptake of HIV/AIDS services in people at high risk for HIV exposure. The study showed that rapid VCT in health facilities and communities was associated with a large increase in HIV-testing uptake and receipt of results with accepatnce by women were more than men (Pottie, Medu, Welch, Dahal, Tyndal, Rader, \& Wells, 2014) whereas on other hand current study was retrospective analysis of the record of VCT services in one region of Oman to analyze the situation on HIV rapid testing and to identify the high risk group. Similarly, in this current study VCT uptake was found to be more among females 990 (70.1\%) as compared to males. RDTs were utilized mainly during outreach activities as compared to the clinical setting in the counselling clinic by the VCT clients through hotline.

According to the WHO Universal Access Progress report 2010, the coverage of HIV testing in the Eastern Mediterranean Region is only 3.6 tests per thousand adult population, including those tests conducted without counselling. The reasons for this are multi-factorial and complex but not necessarily insurmountable. High levels of stigma among both the general public and local communities and, notably, among health care workers, political sensitivities and cultural constraints consistently emerge as barriers to accessing HIV prevention and care services in the Region, (WHO-EMRO, 2011). Due to high prevalence of HIV in few regions of Oman, Ministry of Health Oman launched recently VCT services free of cost in order to detect early HIV infection and to reach out to high risk clients in the community. Although, RDTs are integrated into services in the form of VCT and outreach activities as a first step but confirmation through ELISA and western blot are considered as second screening assay in order to overcome limitations of RDTs for reducing false negative and false positive results. As during current study, it was found that only 1 client was tested positive using RDTs but ELISA and Western Blot as second screening test was performed to confirm the diagnosis of HIV infection. Rapid HIV testing in detection of early HIV infection has the potential to become a valuable and reliable tool despits its shortcomings and disadvnatages and it should be supervised and administered by well trained clinic staff or experinec professionals.

\section{Conclusion}

This study was first of its kind in the country to analyze the situation on HIV rapid testing in Al Buraimi Governorate because of high prevalence of HIV in this region of Oman. Heterosexual was considered as a major risk factor among clients with male predominance and adults aged 15-35 years which is mainly reproductive and high risk age group. So, there is a need of development of risk reduction plan in order to facilitate behavioral change among clients with high risk factor. RDTs were utilized mainly during outreach activities as compared to the clinical setting in the counselling clinic by the VCT clients through hotline. VCT service in the province will offer an entry point for early care and support for those infected with HIV and Prevention of Mother to Child Transmission (PMTCT). Furthermore, such studies in other province of the country to be conducted in order to generalize the results.

\section{Acknowledgments}

The authors sincerely acknowledge the Director of Al Buraimi hospital and Director General of Health Services for their entire support and promoting activities related to VCT in the Governorate.

\section{Authors Contribution}

SRSA composed the manuscript and planned the study; MMU worked on methodology, accomplished the interpretation of results and statistical analysis, AYMDAB did revision with critical review, RK and VKP did the data collection and management.

\section{Ethics Approval}

The study was approved by Regional Research and Ethical Review Committee Directorate General of Health Services Al Buraimi Governorate, Oman.

\section{Competing Interests Statement}

There is no conflict of interest among authors and they have no actual or potential competing financial interests.

\section{References}

Hermez, J., Petrak, J., Karkouri, M., \& Riedner, G. (2010). A review of HIV testing and counseling policies and practices in the Eastern Mediterranean Region. AIDS, 24, 25-32. https://doi.org/10.1097/01.aids.0000386730.56683.e5

HIV management in Oman. (2015). A guide for health care workers ( $3^{\text {rd }}$ ed). Naitional AIDS Program. Department of Communicable Diseases. Directorate General for Disease Surveillance and Control, Minisrty of Health, Oman. Retrieved 25 Oct, 2017 , from 
https://www.moh.gov.om/en/web/directorate-general-of-disease-surveillance-control/resources

Mehra. B., Bhattar, S., Bhalla, P., \& Rawat, D. (2014). Rapid Tests versus ELISA for Screening of HIV Infection: Our Experience from a Voluntary Counselling and Testing Facility of a Tertiary Care Centre in North India. ISRN AIDS, 296840. https://doi.org/10.1155/2014/296840

Pottie. K., Medu, O., Welch, V., P Dahal, G., Tyndal, M., Rader, T., \& Wells, G. (2014). Effect of rapid HIV testing on HIV incidence and services in populations at high risk for HIV exposure: an equity-focused systematic review. $B M J$, 4, e006859. https://doi.org/10.1136/bmjopen-2014-006859

UNAIDS. (2017). Ending AIDS: Progress towards the 90-90-90 targets. Retrieved 22 Oct, 2017, from http://www.unaids.org/en/resources/documents/2017/20170720_Global_AIDS_update_2017

UNAIDS. (2018). Global HIV \& AIDS statistics-July 2018 fact sheet. Retrieved from http://www.unaids.org/en/resources/fact-sheet

World Health Organization [WHO]. (2015). Fact sheet to the WHO consolidated guidelines on HIV testing services.

WHO, UNITAID. (July 2016). Landscape for HIV rapid diagnostic tests for HIV self-testing Report (2nd ed.). Retrieved $20 \quad$ Oct, 2017, from http://www.who.int/hiv/mediacentre/news/hiv-self-testing-landscape-report-2016/en/

WHO-EMRO. (2011). The situation of HIV testing and counselling policies and practices in the Eastern Mediterranean Region. EMRO Technical Publications Series 2011.

\section{Copyrights}

Copyright for this article is retained by the author(s), with first publication rights granted to the journal.

This is an open-access article distributed under the terms and conditions of the Creative Commons Attribution license (http://creativecommons.org/licenses/by/4.0/). 